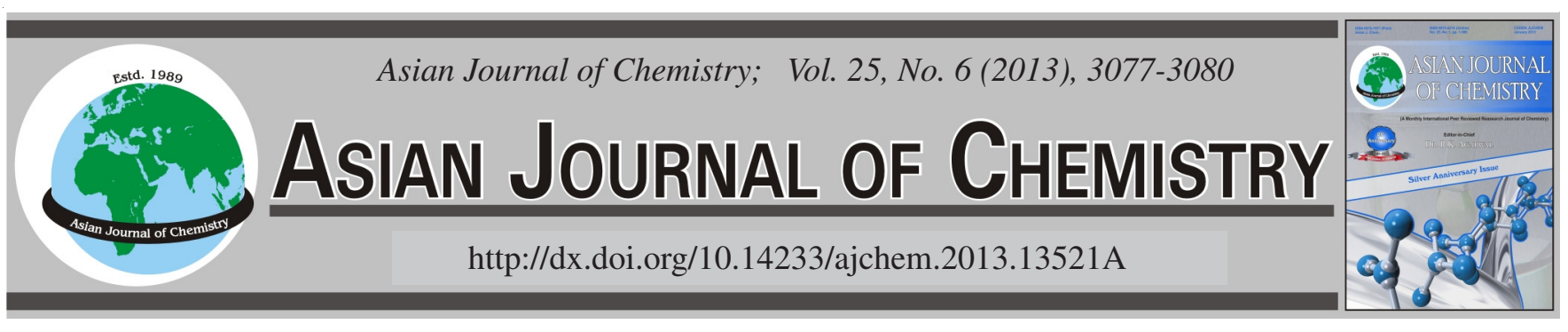

\title{
Poly(vinyl pyrrolidone)-Sodium Dodecyl Sulfate Complex as a Probe to Study the Effect of Benzyl Alcohol on Micellization Behaviour of Sodium Dodecyl Sulfate
}

\author{
XiAoming Chen $^{1,2, *}$ and Lin Ren ${ }^{1}$ \\ ${ }^{1}$ School of Materials and Chemical Engineering, Anhui University of Architecture, Hefei 230601, P.R.China \\ ${ }^{2}$ Anhui Key Laboratory of Advanced Building Materials, Hefei 230601, P.R. China \\ *Corresponding author: Tel: +86 551 3824970, E-mail: chenxmyn@ @otmail.com
}

(Received: 28 January 2012;

Accepted: 12 December 2012)

AJC-12522

Poly(vinyl pyrrolidone)/sodium dodecyl sulfate complex was applied as a probe to study the effect of benzyl alcohol on micellization
behaviour of sodium dodecyl sulfate. It has been found that a critical concentration $\left(\mathrm{c}_{\mathrm{s}}\right)$, exists in the relative viscosity versus benzyl
alcohol concentration plot. As the benzyl alcohol concentration is less than $\left(\mathrm{c}_{\mathrm{s}}\right)$ the relative viscosity of solution containing poly(vinyl
pyrrolidone)/sodium dodecyl sulfate complex increased by adding benzyl alcohol into solution successively. On the other hand, as the
benzyl alcohol concentration is greater than $\mathrm{c}_{\mathrm{s}}$, the relative viscosity of solution containing poly(vinyl pyrrolidone)/sodium dodecyl
sulfate complex decreased by adding benzyl alcohol into solution. The increase and the decrease of the relative viscosity of solution
containing poly(vinyl pyrrolidone)/sodium dodecyl sulfate complex in the presence of benzyl alcohol is just due to the cosurfactant and
the cosolvent effect on micellization behaviour of sodium dodecyl sulfate in aqueous solution. Further experimental results indicate that
$\mathrm{c}_{\mathrm{s}}$ is dependent on both sodium dodecyl sulfate and Poly(vinyl pyrrolidone) concentration.

Key Words: Poly(vinyl pyrrolidone)/Sodium dodecyl sulfate complex, Benzyl alcohol, The relative viscosity.

\section{INTRODUCTION}

Alcohols are often used as additives for surfactant formulations to obtain desired properties. It is well known that the nonionic character of the alcohol leads to a diluted surface charge density in the palisade layer, thereby introducing a decrease in the critical micelle concentration (CMC) of the surfactant $^{1,2}$. This is known as the cosurfactant effect. At higher alcohol concentration the solution is more hydrophobic (the solubility of an ionic surfactant increases) and the dielectric constant of the solvent decreases (the electrostatic repulsion of the ionic headgroups increases) thereby the $\mathrm{CMC}$ of an ionic surfactant increases. This is known as the cosolvent effect ${ }^{1,3,4}$.

Considering that spherical micelles of surfactants and alcohols have low ability to increase the viscosity of solution, the interesting effect of alcohols on micellization behaviour of an ionic surfactant could not be effectively detected by viscosity measurements. However it is well known that the complex of macromolecules with surfactants can be studied effectively and conveniently by the viscosity measurements ${ }^{5-15}$.

In this study we present a new technique by which the cosurfactant and the cosolvent effect of benzyl alcohol on micellization behaviour of sodium dodecyl sulfate in aqueous solution can be detected effectively. According to the new tech- nique the macromolecules were selected to form complex with surfactant molecules. As the alcohol is adding into the solution containing the complex of macromolecules and surfactants successively, the amount of polymer-bound surfactant changes, thereby the conformation of polymer and thus the solution viscosity changes significantly. As a result, the effect of benzyl alcohol on micellization behaviour of sodium dodecyl sulfate can be detected by viscosity measurement. On such an occasion the polymer/surfactant complex acts essentially as a viscosity probe. Having selected poly(vinyl pyrrolidone)/sodium dodecyl sulfate complex as the probe, the cosurfactant and the cosolvent effect of benzyl alcohol on micellization behaviour of sodium dodecyl sulfate was studied thoroughly.

\section{EXPERIMENTAL}

Poly(vinyl pyrrolidone) sample employed in this work is a commercial product of Sigma Chemical Company. The average molecular weight is $3.6 \times 10^{5}$. Anionic surfactant sodium dodecyl sulfate (purity $>99.9 \%$ ) is purchased from Xuzhou (China) second chemical company and benzyl alcohol (purity > $99 \%$ ) is from Sinopharm Chemical Reagent Co. Ltd. All the samples were used as received without any further purification. De-ionized distilled water was used in all experiments here. 
All viscosity measurements were carried out using a conventional Ubbelohde capillary viscometer(inner diameter $\phi=0.55 \mathrm{~mm})$ at $30 \pm 0.1^{\circ} \mathrm{C}$ maintained with a thermostatic water bath. Measurements were initiated after approximately 5-10 min equilibrium time. Each flow time was determined by repeating at least three time measurements. The precision of the measurements was $0.01 \mathrm{sec}$ and the reproducibility was $0.2 \mathrm{sec}$. The relative viscosity was calculated from $\eta_{\mathrm{r}}=\mathrm{t} / \mathrm{t}_{0}$ with $t$ and $t_{0}$ the flow time of the solution and the pure water, respectively, neglecting the difference of the density between the solution and the solvent as performed in previous studies ${ }^{16-20}$.

The experimental procedures were as follows. Two stock solutions were prepared, both containing the desired concentrations of poly(vinyl pyrrolidone) and sodium dodecyl sulfate. A high concentration of benzyl alcohol was also included in one of the solutions. Known quantities of the stock solutions were mixed to give any desired concentration of benzyl alcohol. This procedure was used for all experiments in which it was desired to vary the benzyl alcohol concentration systematically.

\section{RESULTS AND DISCUSSION}

Fig. 1 shows the relative viscosity of sodium dodecyl sulfate both in pure water and in the aqueous poly(vinyl pyrrolidone) solution at $30^{\circ} \mathrm{C}$. It can be seen that two critical concentrations, namely $\mathrm{c}_{1}$ and $\mathrm{c}_{2}$, exist for sodium dodecyl sulfate in presence of the poly(vinyl pyrrolidone). The concentration $\mathrm{c}_{1}$, known as the critical aggregation concentration (CAC), indicates sodium dodecyl sulfate has bound to poly(vinyl pyrrolidone) chains to form poly(vinyl pyrrolidone)/ sodium dodecyl sulfate complex in the formation of micellelike clusters ${ }^{21}$. Obviously, the electric repulsion between polymer-supported micelles will produce the expansion of poly(vinyl pyrrolidone) chains in solution. As a result, the relative viscosity $\eta_{\mathrm{r}}$ increases with increasing concentration of sodium dodecyl sulfate (Fig. 1). The maximum of $\eta_{\mathrm{r}}$ at the critical concentration $\mathrm{c}_{2}$ indicates the saturation of bound micelles to polymer chains and the subsequent decrease of the relative viscosity on further addition of sodium dodecyl sulfate is due to the screening of charge interactions by free micelles of sodium dodecyl sulfate in solution. Fig. 1 showed that to applying poly(vinyl pyrrolidone)/sodium dodecyl sulfate as the viscosity probe, the desired concentration of sodium dodecyl sulfate should be less than $\mathrm{c}_{2}$ whereas greater than $c_{1}$. In present experiment, the concentration of sodium dodecyl sulfate was selected as $6 \mathrm{mM}$.

Fig. 2 shows the plot of the relative viscosity $\eta_{\mathrm{r}}$ of benzyl alcohol in poly(vinyl pyrrolidone)/sodium dodecyl sulfate solution, poly(vinyl pyrrolidone) solution, sodium dodecyl sulfate solution and pure water respectively at $30^{\circ} \mathrm{C}$. It can be seen that the relative viscosity of benzyl alcohol in poly(vinyl pyrrolidone) solution, sodium dodecyl sulfate solution and pure water only increase slightly with the increasing concentration of benzyl alcohol. More probably, the results indicate that the effect of the interaction of benzyl alcohol and sodium dodecyl sulfate [or poly(vinyl pyrrolidone] upon the viscosity of solution on such an occasion is negligible. On the other hand, in poly(vinyl pyrrolidone)/sodium dodecyl sulfate solution, the relationship between the relative viscosity and the concentration of benzyl alcohol is quite different.

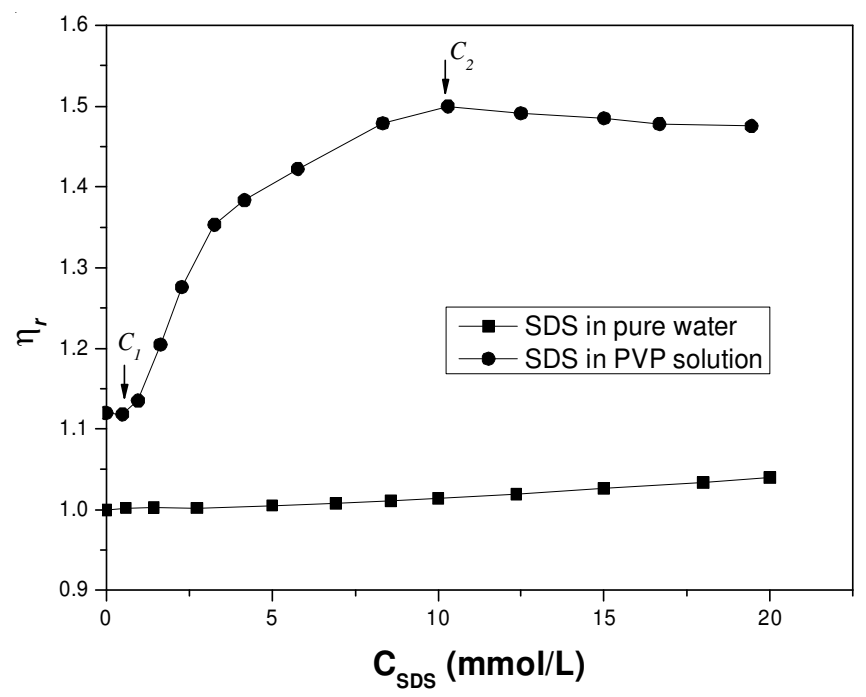

Fig. 1. Relative viscosity $\eta_{\mathrm{r}}$ of sodium dodecyl sulfate in both water and poly(vinyl pyrrolidone) solution at $30{ }^{\circ} \mathrm{C}$. The concentration of poly(vinyl pyrrolidone) is $0.001 \mathrm{~g} / \mathrm{mL}$

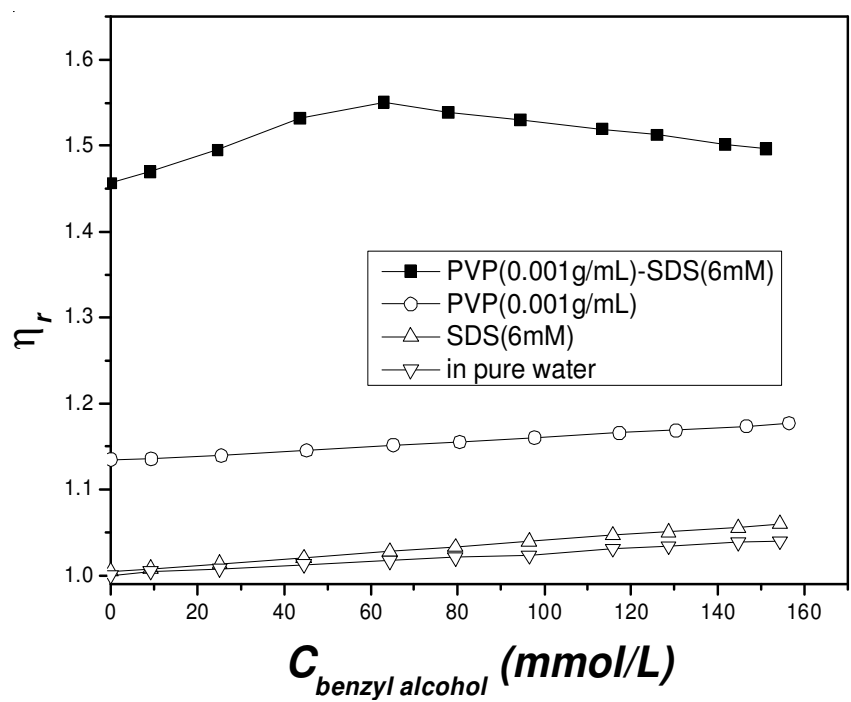

Fig. 2. Relative viscosity $\eta_{\mathrm{r}}$ of benzyl alcohol in PVP/SDS solution, poly(vinyl pyrrolidone) solution, sodium dodecyl sulfate solution and pure water respectively at $30^{\circ} \mathrm{C}$.

Fig. 2 showed that the relative viscosity of benzyl alcohol in poly(vinyl pyrrolidone)/sodium dodecyl sulfate solution increases with increasing benzyl alcohol concentration. However, as the concentration of benzyl alcohol is greater than $\mathrm{c}_{\mathrm{s}}$, the relative viscosity of benzyl alcohol in poly(vinyl pyrrolidone)/sodium dodecyl sulfate solution decreases with increasing benzyl alcohol concentration. The reasonable interpretation is as follows. Benzyl alcohols penetrate into the palisade layer of polymer-bound sodium dodecyl sulfate micelles and the nonionic character of benzyl alcohol leads to a diluted surface charge density in the palisade layer. As a result more free sodium dodecyl sulfate molecules could aggregate with the polymer-bound micelles. That is to say the amount of polymer-bound sodium dodecyl sulfate molecules increases resulting in the expansion of poly(vinyl pyrrolidone) 
chains. Such results reflect the cosurfactant effect of benzyl alcohol on the micellization behaviour of sodium dodecyl sulfate. As the benzyl alcohol concentration increases, the solution is more hydrophobic (the solubility of an ionic surfactant increases) and the dielectric constant of the solvent decreases (the electrostatic repulsion of the ionic headgroups increases), thereby the polymer-bound micelles become destroyed. This is known as the cosolvent effect. As can be seen in Fig. 2 when the benzyl alcohol concentration is greater than critical concentration the cosolvent effect dominates and thereby it introduces the decrease of the amount of polymerbound sodium dodecyl sulfate molecules. As a result the poly(vinyl pyrrolidone) chains shrink gradually.

Fig. 3 shows the relative viscosity $\eta_{\mathrm{r}}$ of benzyl alcohol in poly(vinyl pyrrolidone)/sodium dodecyl sulfate solution at $30{ }^{\circ} \mathrm{C}$. In Fig. 3 the concentration of poly(vinyl pyrrolidone) is fixed to be $0.001 \mathrm{~g} / \mathrm{mL}$, just the same as indicated in Fig. 2. On the other hand, the concentration of sodium dodecyl sulfate is selected to be $4 \mathrm{mM}, 6 \mathrm{mM}$ and $8 \mathrm{mM}$, respectively. From Fig. 3 it can be seen that with the increase of sodium dodecyl sulfate concentration critical concentration decreases accordingly. This is due to the reason that the poly(vinyl pyrrolidone) chains is gradually saturated with sodium dodecyl sulfate when the sodium dodecyl sulfate concentration increases. Thus the sodium dodecyl sulfate molecules that could be bound to poly(vinyl pyrrolidone) chains decreases. As a result critical concentration for benzyl alcohol is lower. Fig. 4 also shows the relative viscosity $\eta_{\mathrm{r}}$ of benzyl alcohol in poly(vinyl pyrrolidone)/sodium dodecyl sulfate solution at $30^{\circ} \mathrm{C}$. In Fig. 3 the concentration of sodium dodecyl sulfate is fixed to be $6 \mathrm{mM}$ but the concentration of poly(vinyl pyrrolidone) is selected to be $0.0008 \mathrm{~g} / \mathrm{mL}, 0.001 \mathrm{~g} / \mathrm{mL}$ and $0.0012 \mathrm{~g} / \mathrm{mL}$, respectively. Considering that at constant sodium dodecyl sulfate concentration the more poly(vinyl pyrrolidone) chains the solution has, the more polymer-bound sodium dodecyl sulfate molecules could exist. As a result critical concentration for benzyl alcohol is higher when the poly(vinyl pyrrolidone) concentration increases, verifying our assumption completely.

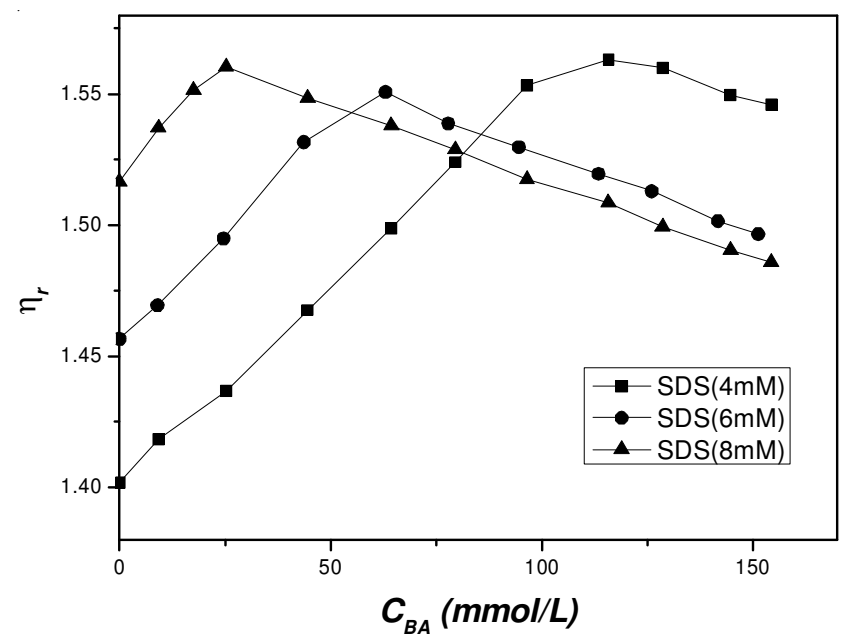

Fig. 3. Relative viscosity $\eta_{\mathrm{r}}$ of benzyl alcohol in poly(vinyl pyrrolidone)/ sodium dodecyl sulfate (PVP/SDS) solution at $30^{\circ} \mathrm{C}$. The concentration of poly(vinyl pyrrolidone) is fixed to be $0.001 \mathrm{~g} / \mathrm{mL}$ whereas the concentration of sodium dodecyl sulfate is selected to be $4 \mathrm{mM}$, $6 \mathrm{mM}, 8 \mathrm{mM}$ respectively

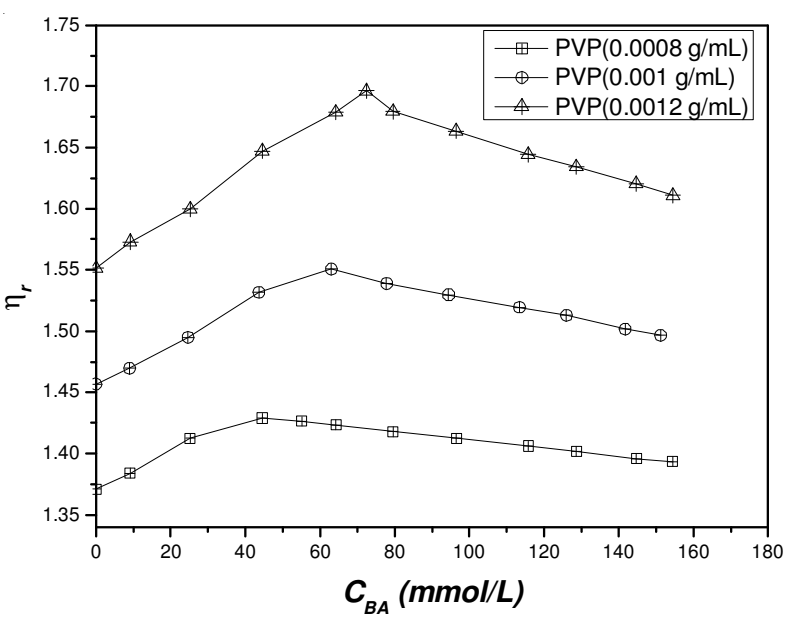

Fig. 4. Relative viscosity $\eta_{\mathrm{r}}$ of benzyl alcohol in PVP/SDS solution at $30^{\circ} \mathrm{C}$. The concentration of sodium dodecyl sulfate is fixed to be $6 \mathrm{mM}$ whereas the concentration of poly(vinyl pyrrolidone) is selected to be $0.0008 \mathrm{~g} / \mathrm{mL}, 0.001 \mathrm{~g} / \mathrm{mL}$ and $0.0012 \mathrm{~g} / \mathrm{mL}$ respectively

\section{Conclusion}

We have shown that poly(vinyl pyrrolidone)/sodium dodecyl sulfate complex can be applied as a probe to study the effect of benzyl alcohol on micellization behaviour of sodium dodecyl sulfate in aqueous solution. Our technique is based on the design that the cosurfactant and the cosolvent effect of benzyl alcohol on micellization of sodium dodecyl sulfate will change the amount of the polymer-bound sodium dodecyl sulfate molecules and the conformation of the polymer chains will change accordingly. On such an occasion, the viscosity of solution containing poly(vinyl pyrrolidone)/sodium dodecyl sulfate complex will change significantly. As a result the effect of benzyl alcohol on micellization behaviour of sodium dodecyl sulfate can be detected by viscosity measurement. poly(vinyl pyrrolidone)/sodium dodecyl sulfate complex, as presented in our new technique, acts essentially as a viscosity probe. Our experimental results indicate that a critical concentration, namely critical concentration, exists in the relative viscosity versus benzyl alcohol concentration plot. As the benzyl alcohol concentration is less than critical concentration, the relative viscosity of solution containing poly(vinyl pyrrolidone)/sodium dodecyl sulfate complex increased by adding benzyl alcohol into solution successively. On the other hand, as the benzyl alcohol concentration is greater than critical concentration, the relative viscosity of solution containing poly(vinyl pyrrolidone)/sodium dodecyl sulfate complex decreased by adding benzyl alcohol into solution. The increase and the decrease of the relative viscosity of solution containing poly(vinyl pyrrolidone)/sodium dodecyl sulfate complex in the presence of benzyl alcohol is just due to the cosurfactant and the cosolvent effect on micellization behaviour of sodium dodecyl sulfate in aqueous solution. Further experimental results indicate that critical concentration is dependent on both sodium dodecyl sulfate and poly(vinyl pyrrolidone) concentration.

\section{ACKNOWLEDGEMENTS}

The project was supported by the Doctor Research Foundation of Anhui University of Architecture (K02541), the 
Students Scientific Innovation Program of Anhui University of Architecture(C11039), the Postdoctoral Science Foundation of Anhui University of Architecture (20110101) and Key Project of Anhui Provincial College Natural Science (KJ2011A070).

\section{REFERENCES}

1. P.C. Griffiths, N. Hirst, A. Paul, S.M. King, R.K. Heenan and R. Farley, Langmuir, 20, 6904 (2004)

2. S. Cinelli, G. Onori and A. Santucci, Colloids Surf. A, 160, 3 (1999).

3. J.B. Huang, M. Mao and B.Y. Zhu, Colloids Surf. A, 155, 339 (1999).

4. H. Gharibi, B.M. Razavizadeh and A.A. Rafati, Colloids Surf. A, 136, 123 (1998).

5. J. Fundin, P. Hansson, W. Brown and I. Lidegran, Macromolecules, 30, 1118 (1997)

6. T. Aubry, M. Moan, J.F. Argillier and A. Audibert, Macromolecules, 31, 9072 (1998).

7. S. Panmai, R.K. Prudhomme and D.G. Peiffer, Colloids Surf. A., 147, 3 (1999).

8. K.Y. Mya, A.M. Jamieson and A. Sirivat, Langmuir, 16, 6131 (2000).
9. E. Hoff, B. Nyström and B. Lindman, Langmuir, 17, 28 (2001).

10. X. Zheng and W. Cao, Eur. Polym. J., 37, 2259 (2001).

11. D. Zanette, V. Soldi, A.P. Romani and M.H. Gehlen, J. Colloid Interf. Sci., 246, 387 (2002).

12. S. Guillot, M. Delsanti, S. Désert and D. Langevin, Langmuir, 19, 230 (2003).

13. N. Plucktaveesak, A.J. Konop and R.H. Colby, J. Phys. Chem. B, 107, 8166 (2003).

14. P.C. Griffiths, N. Hirst, A. Paul, S.M. King, R.K. Heenan and R. Farley, Langmuir, 20, 6904 (2004)

15. N. Jain, S. Trabelsi, S. Guillot, D. McLoughlin, D. Langevin, P. Letellier and M. Turmine, Langmuir, 20, 8496 (2004).

16. H. Yang, Y. Yan, P. Zhu, H. Li, Q. Zhu and C. Fan, Eur. Polym. J., 41, 329 (2005).

17. H. Yang, S. Zhang, H. Liu, X. Xu, J. Zhang and P. He, J. Mater. Sci. Lett., 40, 4645 (2005).

18. X. Chen, H. Yang, X. You, P. Zhu and P. He, Chin. J. Polym. Sci., 24, 437 (2006).

19. J. Li, H. Li, H. Yang, P. Zhu and P. He, Chin. J. Polym. Sci., 26, 31 (2008).

20. Y. Yan, H. Li, H. Yang, J. Qian, P. Zhu and P. He, Chin. J. Chem. Phys., 21, 169 (2008)

21. E.D. Goddard, J. Am. Oil Chem. Soc., 71, 1 (1994). 